\title{
User Perception of Algorithmic Digital Marketing in Conditions of Scarcity
}

\author{
Veronika Pavlidou ${ }^{10000-0003-2936-9867]}$, \\ Jahna Otterbacher ${ }^{1,2[0000-0002-7655-7118]}$, and \\ Styliani Kleanthous ${ }^{1,2}$ [0000-0003-1594-1340] \\ 1 Open University of Cyprus, Latsia, CYPRUS \\ 2 CYENS Centre of Excellence, Nicosia, CYPRUS
}

\begin{abstract}
Digital Marketing, and specifically, targeted marketing online is flourishing in recent years, and is becoming evermore precise and easy to implement, given the rise of big data and algorithmic processes. This study assesses users' perceptions regarding the fairness in algorithmic targeted marketing, in conditions of scarcity. This is increasingly important because as more decisions are made by data-driven algorithms, the potential for consumers to be treated unfairly by marketers grows. Awareness of users' perceptions helps to create a more open, understandable and fair digital world without negative influences. Also, it may help both marketers and consumers to communicate effectively.
\end{abstract}

Keywords: digital marketing · users' perceptions · fairness in algorithmic processes $\cdot$ microtargeting $\cdot$ scarcity messages.

\section{Introduction}

Businesses, and especially brands, have long embraced technology to remain competitive and deliver the best customer services. "Cognitive technologies" is a more recent industry term for narrow $\mathrm{AI}^{1}$, implying that the technologies take the automation to a new level of "human-like" behaviours. Algorithmicmediated, targeted marketing online, based on models created via machine learning (ML), has greatly influenced marketing practices. Today, most people conduct online research before making a purchase, and this fundamental change in buying behaviour forces marketers to adapt their business marketing strategies for the digital age. Marketers (i.e., advertisers) always try to reach their target audience based on demographics (gender, age, race, ethnicity), preferences, etc. and by using cognitive biases (e.g, scarcity bias), that influence potential consumer behaviour and decisions in order to increase sales. It is generally accepted

\footnotetext{
${ }^{1}$ Artificial intelligence systems focused on a singular or limited task.
} https://deepai.org/machine-learning-glossary-and-terms/narrow-ai 
that AI systems have many potential benefits for business, the economy, and for tackling society's most pressing social challenges, including the mitigation of inequality. But that will only be possible if people trust these systems to produce 'fair results' - or at least, results that are explained to the user.

As (O'Neil, 2016) states "no model can include all the real world's complexity or the nuance of human communication." Thus, new issues have come up regarding the fairness of algorithmic decisions. According to IEEE Global Initiative on Ethics of Autonomous and Intelligent Systems document (2019), intelligent systems must respect human rights, freedoms, human dignity, and cultural diversity. They must be verifiably safe and secure throughout their operational lifetime and stakeholders should prioritize human well-being (e.g. mental health, emotions, sense of themselves, their autonomy, etc.) as an outcome in all system designs, using the best available and widely accepted well-being metrics as their reference point. And since machine learning algorithms used to automate decisions already affect individuals, businesses and other organizations globally, concerns regarding the fairness of the algorithmic decisions raised by the expert community are numerous. They include the lack of algorithmic fairness (leading to discriminatory practices such as racial and gender biases), content personalisation resulting in partial information asymmetry (e.g 'filter bubble'), lack of transparency, the infringement of user privacy, and potential user manipulation (Lepri, Oliver, Latouze, Pentland, \& Vinck, 2017).

It is important for marketers to understand consumers' perceptions, and specifically fairness perceptions, to determine the factors that influence consumers to make purchase decisions. Moreover, the concept of fairness is essential to the relationship between consumers and marketers. Currently, we present an online study that aims to identify the existence of concerns about the targeted marketing online in conditions of scarcity. The study works towards understanding the perceptions of users, considering their level of awareness of issues related to bias and unfairness in algorithmic targeted marketing. Moreover, it examines the level of tailored-made ads with scarcity with which users are comfortable. The more people made aware of how these algorithms operate, the better chance there is to foster trust in these systems, as informed users will be in position to make an assessment as to whether they are being treated fairly or not.

\section{Related Work}

\subsection{Targeted marketing online}

Microtargeting techniques are really powerful, but can be potentially dangerous. (Howard, 2006). As (Kleinberg, Ludwig, Mullainathan, \& Sunstein, 2019) state "algorithms are fundamentally opaque, not just cognitively but even mathematically." In other words, these processes are typically "black boxes," with the 
model of the consumer typically being constructed algorithmically (e.g., by exploiting her or his online behaviours to infer demographic characteristics and preferences) (Bayer, 2020). For example, Wing's model of "Data and ML relation" (Wing, 2018) shows that training data are input to a machine learning algorithm to produce a model that can classify the data subject (i.e., consumer) or make predictions. If the data is somehow biased, the algorithm will be affected, implying a biased model that produces biased results.

One of the upcoming trends in the E-Commerce industry is "contextual and programmatic advertising," which aims to provide the right audience with the right ad at the right moment. These techniques are expected to use huge amounts of data to identify target customers (Big Data in E-commerce: Global Markets, 2019). Marketers create ads based on their users' collected datasets, comprising observations on their demographic, historical, current, geographic location, etc. After publishing an ad, the contextual advertising system (e.g., Google's AdSense) then directs the ads, through keywords, to the right websites.

\subsection{Users' perceptions of fairness in algorithmic processes}

While targeted marketing has generally been viewed as beneficial to both consumers and marketers, there are concerns raised of how consumers perceive and reason about fairness in algorithmic processes. According to (Lee, 2018), how users perceive, and process issues of FATE (Fairness, Accountability, Transparency and Ethics) is considered more important than the technical properties/qualities of the algorithmic processes driving targeted ads, such as accuracy and predictability. As (Shin \& Park, 2019) argued, users expect algorithms to offer accurate, convenient, and credible results; in other words, users are inclined to trust the system because they know how the data are analyzed and thus how recommendations are generated. Moreover, users with higher levels of trust were observed to be more likely to see algorithms as fair, accurate, and transparent, while trust moderating the relationship between FATE and satisfaction. However, public understanding is limited by a technical barrier as well as economic factors, with many algorithmic processes protected as trade secrets.

\subsection{Persuasive marketing techniques}

Consumer cognitive biases help marketers to develop successful campaigns. This strategy is known as cognitive marketing. "Cognitive marketing is one of the best ways to connect with a customer on a personal level. It essentially uses what people are already thinking about in a positive way to give a brand a position that reflects the customer's position. This helps the customer to see a similarity between themselves and the brand, helping to form a strong connection" (What 
is Cognitive Marketing and Why Should You Be Using It?, 2017). For example, companies such as Dove and its 2004 "campaign for real beauty", tried to connect with a customer by reducing the consumer's gap between the actual and ideal self or the Always company and its 2014 campaign "\#likeagirl", with a very powerful meaning. Cognitive marketing campaigns focus on full targeting (i.e., demographic, geographic) and use different cognitive biases to be effective as possible. However, the most well-known is a scarcity bias.

Scarcity bias or effect technique in Digital Marketing. The definition of Scarcity can be found in early marketing literature, and refers to a commodity's unavailability. Scarcity may be operationalized as: (1) limits on the supply of a commodity; (2) costs of acquiring, or of providing, a commodity; (3) restrictions limiting possession of a commodity, and/or (4) delays in providing a commodity (Brock, 1968). Commodities refer to any marketable goods or services such as promotions, offers, events, etc. There are several types of scarcity: supply-caused scarcity (Cialdini, 2008), demand-caused scarcity and time scarcity (Gierl \& Huettl, 2010), exclusivity or uniqueness (Fromkin, 1970). Demand-caused scarcity and time scarcity can be defined as the presence of limited resources and competition on the demand side (e.g., not enough for two people) (Mittone \& Savadori, 2009). This type of scarcity is often used in promotion by big brands' "limited edition products" (Aggarwal, Jun \& Huh, 2011). In contrast, Exclusivity is a specific type of scarcity, in which only a selected group of people receives a promotion (Broeder \& Derksen, 2018) or Uniqueness can be defined as a humans' need to feel that they are not too similar to others (Schumpe \& Erb, 2016).

As (Mittone \& Savadori, 2009) state, scarcity does work as an attractor as humans place a higher value on an object that is scarce. As (Aggarwal, Jun, \& Huh, 2011) explain, scarcity creates a sense of urgency among buyers that results in increased quantities purchased, shorter searches, and greater satisfaction with the purchased products. Today's world can be described as a time of unprecedented abundance, yet scarcity persists, and it is used in digital marketing as a tool for boosting consumption, creating wants and needs for the product or service that marketers would like to promote. As (O'Neil, 2016) characteristically described "lead aggregators push people toward needless transactions... the data-driven algorithms, while producing revenue for search engines, lead aggregators, and marketers, is a leech on the economy as a whole". Announcements such as "In high demand", "Two seats left at this price!" or "Sale, by Invitation only" represent scarcity use in digital marketing. Such tactics are commonly found on platforms such as booking.com, amazon.com, ebay.com etc.

Perceptions of persuasive marketing techniques. On the one hand, a scarcity bias (effect) in digital marketing is likely to elicit positive thoughts about the product (Gierl \& Huettl, 2010). And on the other, it can also provoke a negative thought, such as suspicion about marketers' manipulative intent (i.e., persuasive attempt). For example, it is possible for marketers to artificially 
restrict the quantity of a product being offered in a given retail outlet or sales territory, and thus send a false signal of popularity among consumers. In this way, according to (Gupta, 2013), scarcity communicated by the retailer threatens consumers' freedom. Another example of an ethical issue is that when consumers compete against one another, the seller stands to benefit from such competition (Aggarwal, Jun \& Huh, 2011). Clearly, persons should not be forced, tempted, or seduced into performing actions solely for the benefit of a third party (Becker, 2019). Generally, in the digital marketing literature, it is clear that controversial ethical issues arise when tactics such as scarcity are employed by marketers.

Effectiveness of Scarcity in Digital marketing The framework of (Xiaohui, Feng, \& Pattarin, 2020) indicates that the effective use of product scarcity in marketing depends on a combination of consumer characteristics, types of scarcity, and types of product, which results in different impacts on consumers. Also, as (Broeder \& Derksen, 2018) state, the effectiveness of scarcity may differ depending on the type of product and advertising context and they found that even though advertisements are not likable, they can be effective, indicating that the likability of advertisements is not necessarily related to effectiveness. Similarly, (Khoa Trinh, 2014) notes that the ease of searching for alternative online deals may change the effectiveness of scarcity messages.

According to (Lynn, 1991) scarcity tactics are more effective when targeted at consumers who possess greater than average needs for uniqueness. Such targeting would be facilitated by an understanding of the demographic, lifestyle, and other characteristics of high need-for-uniqueness individuals. Also, as (Lynn, 1991) states, scarcity has an effect only when subjects had been primed to think about the price implications of the scarcity. In other words, scarcity enhances the value (or desirability) of anything that can be possessed (Brock,1968). Thus, marketers can increase the perceived value of products, services, and promotions by manipulating the perceived scarcity of the offerings.

Lastly, microtargeting doesn't act as a magic bullet in consumer persuasion but is not pointless for advertisers or harmless for consumers (Winter, Maslowska, Vos, 2021). The previous studies propose to investigate the effectiveness of scarcity appeals in the context of consumer-choice setting and behavioral retargeting. Also, the fairness variable is suggested to be investigated since it is essential to the relationship between consumers and marketers.

\section{Methodology and Research Questions}

To explore users' perceptions regarding fairness in algorithmic processes that are commonly used in e-commerce contexts and in conditions of scarcity, a twophase, mixed-methods study was conducted, in order to answer the following research questions (RQ): RQ1. How do users perceive algorithmically mediated 
digital marketing? RQ2. How do consumers perceive and respond to Scarcity in algorithmically mediated digital marketing? RQ3. What are users' perceptions about the fairness in use of scarcity in algorithmic micro-targeting.

In the first phase of the study, we conducted an online experiment, involving a simulated e-commerce context, which participants were asked to visit. We then assessed their perceptions and feelings surrounding the use of algorithmic microtargeting, via a questionnaire. In the second phase, follow-up interviews were conducted with five participants, to explore more in-depth their views towards these practices. The participants of the study were recruited at the first author's college. Our research protocol and informed consent materials received ethical approval from [redacted for blind review.

Phase 1: Online Experiment. Our online experiment was implemented in three stages: a pre-experiment questionnaire (PQ1), participation in a simulated, online browsing experience, and finally, a post-experiment questionnaire (PQ2) (see Table 1). PQ1 (consisting of 16 questions) consisted of two parts 1) demographics of the respondent 2) shopping preferences. After participants completed PQ1, two types of ads were designed according to the majority's reported preferences. These ads were placed in between the text as advertising banners on the specific webpage designed for research purposes (i.e., simulated browsing experience), where participants had to sign in. Participants were then directed to the research website. First, they were told that they would be asked to read a text concerning higher education issues. Following that, they would be prompted to complete the post-questionnaire (PQ2) that would take them approximately 10 minutes. PQ2 (consisting of a series of 32 seven-point Likert item questions, with possible responses ranging from "strongly disagree" (1), "neutral" (4), to "strongly agree" (7)) was implemented in three sections: 1) confirmation that the article was read by participants 2) assessing microtargeting concerns 3) assessing their fairness and scarcity perceptions.

Although our experiment aimed to assess participants' views towards the practice of microtargeting, it is also important to understand the behaviour of the participants on the specific webpage, during the experiment. Thus, a website tracking tool has been used. The analysis was focused on the two main points: i) whether participants observed the ads on the page or not and ii) whether participants clicked on the ads or not. Per the responses to PQ2 [Q1-5] we found that most participants ( $\mathrm{N}=44 ; 88 \%$ ) noticed the ads on the page and $47 \%$ believed that ads were tailored to their preferences. Moreover, $65 \%$ found the ads interesting because of the offer and $50 \%$ because of the product.

Participants (Analysis of PQ1). 57 questionnaires in total were returned to the researchers; 50 of these were complete and valid, and the participants agreed to continue to the online experiment. Table 2 presents the demographic attributes of the 50 English-speaking study participants. As can be observed, the participants 
Table 1. Post-Questionnaire (PQ2).

\begin{tabular}{|c|c|}
\hline $\mathrm{a} / \mathrm{a}$ & SECTION 1 confirmation that the article was read by participants \\
\hline 1 & The article you just read was about \\
\hline 2 & On the page was an ad/s for (choose all that apply) \\
\hline 3 & I find the ad interesting because of the product \\
\hline 4 & I find the ad interesting because of the offer \\
\hline & The ad is tailored to my preferencest \\
\hline $\mathrm{a} / \mathrm{a}$ & SECTION 2 microtargeting concerns \\
\hline & When I use social media, I prefer Facebook \\
\hline 7 & I like that Facebook connects me with my friends and with friends of my friends \\
\hline & I like that Facebook connects people and events \\
\hline & I like that Facebook connects photos and People \\
\hline 10 & I like that Facebook ranks the post each user sees \\
\hline 11 & I like that Facebook picks and chooses the ads it thinks users will be interested in seeing \\
\hline 12 & I like that Facebook has access to my activity when I use the social network \\
\hline 13 & I like that Facebook has access to my activity when I visit other websites \\
\hline 14 & I am aware of the consequences of being tracked online \\
\hline 15 & I have no concerns regarding privacy or ethical issues \\
\hline $\mathrm{a} / \mathrm{a}$ & SECTION 3 fairness and scarcity perceptions \\
\hline 16 & $\begin{array}{l}\text { While browsing online, I find that there are many advertisements with messages } \\
\text { like Buy Now!, Only one left!, Today' offer etc }\end{array}$ \\
\hline 17 & When I see the scarcity phrases, I feel that I have to buy this product \\
\hline & When I find ads with scarcity phrases, I tend to ignore them \\
\hline 19 & While shopping online, I find that the products of my interest are often scarce \\
\hline 20 & I like online ads tailored to my preferences \\
\hline 21 & I don't trust the online ads with offers \\
\hline 22 & When I see online ads, I have a feeling that my personal information is taken without permission \\
\hline 23 & When I see online ads, I feel manipulated by the advertiser \\
\hline 24 & I got nervous by seeing the online ads \\
\hline 25 & When I see online ads, I feel that marketers intentionally created scarcity \\
\hline 26 & I think it is not fair to use scarcity to convince me to purchase the product \\
\hline 27 & While browsing online, I develop a desire to buy them immediately \\
\hline 28 & While browsing online, I buy things offered for sale, even I can't afford them \\
\hline 29 & While browsing online, offers and recommendations induce me to do just the \\
\hline 30 & While browsing online, I don't want to run the risk missing out on offers \\
\hline 31 & While browsing online, I become frustrated when I am unable to get my preferred choice \\
\hline 32 & While browsing online, I would be upset if I missed buying some products of interest \\
\hline 33 & I often feel a regret after shopping online \\
\hline 34 & I feel guilty that I can't control my spending \\
\hline
\end{tabular}

are mostly Undergraduate-level (BSc) students from [redacted for blind review] $(\mathrm{N}=30 ; 60 \%)$ and [redacted] $(\mathrm{N}=12 ; 24 \%)$, female $(\mathrm{N}=32 ; 64 \%)$ and male $(\mathrm{N}=18$; $36 \%)$ of age $18-29(\mathrm{~N}=34 ; 68 \%)$. In terms of their interests, they like to listen to music $(\mathrm{N}=33 ; 66 \%)$, watch movies $(\mathrm{N}=27 ; 54 \%)$, and use social media $(\mathrm{N}=25$; $50 \%)$. Many of them $(\mathrm{N}=27 ; 54 \%)$ report spending up to 6 hours per day online for entertainment and only $(\mathrm{N}=4 ; 8 \%)$ more than 6 hours online.

Table 2. Demographic profiles of Phase 1 participants $(\mathrm{N}=50)$.

Survey Participants
\begin{tabular}{|l|l|l|l|}
\hline Variables & Category & $\mathbf{N}$ & $\%$ \\
\hline Gender & Male & 18 & $36 \%$ \\
& Female & 32 & $64 \%$ \\
\hline Marital status & Single & 30 & $53 \%$ \\
& Married & 10 & $20 \%$ \\
& Divorced & 4 & $7 \%$ \\
& Engaged/in a relationship & 6 & $10 \%$ \\
\hline Country & Cyprus & 30 & $60 \%$ \\
& Nepal & 12 & $24 \%$ \\
& India & 4 & $8 \%$ \\
& Other & 4 & $8 \%$ \\
\hline \hline
\end{tabular}


Phase 2: Interviews. Semi-structured interviews (IQ3) consisted of opinion, values and feeling questions aimed at understanding the cognitive and interpretive processes of people's opinions, judgments, values, and feeling responses of people to their experiences and thoughts. Specifically, the goals was to assess i) perceptions on use of algorithmic micro-targeting in online advertising ii) perceptions of its effects iii) perceptions of fairness in use of scarcity in algorithmic micro-targeting. The interview participants were recruited through a request for interview participation sent by email. Before starting (IQ3) the Informed Consent Forms with audio recording use permission were completed. Table 3 presents the demographic attributes of the 5 interview participants.

Table 3. Demographic profiles of Phase 2 participants $(\mathrm{N}=5)$.

Interview Participants
\begin{tabular}{|l|l|l|}
\hline Variables & Category & N \\
\hline Gender & Male & 3 \\
& Female & 2 \\
\hline Country & Cyprus & 5 \\
\hline Age & $18-25$ & 3 \\
& $40-50$ & 2 \\
\hline \hline
\end{tabular}

\subsection{Data analysis}

Both quantitative (PQ2) and qualitative (IQ) data resulted from the study. For the responses to the questionnaire items, we consider the distribution of responses on the Likert item questions. To examine the degree of association between responses across items we use Correlation Analysis (Spearman's r). For now, we do not present a complete analysis of the interview data but use it to enrich the findings stemming from the quantitative data / PQ2.

\section{Analysis}

\section{RQ1. How do users perceive algorithmically mediated digital marketing?}

Most participants prefer to use Facebook $(62 \%)$ and generally like the way the Facebook algorithm works [Q6-10]. Also, all interviewees reported using Facebook and generally also like the way the algorithm of Facebook works. Although $40 \%$ of participants like the Facebook post ranking algorithm, 16\% do not know about it [Q10] and only 34\% like how Facebook picks and chooses the ads for each user [Q11]. The following quotes from interviews illustrate these findings: "I would prefer to have control on what I want to see or not online, but I believe 
that it is too complicated. Sometimes, I intentionally hide the ads just to "disorientate' the algorithm." (interviewee 48, male). In other hand, "I don't know how Facebook works and it is not so important to me" (interviewee 21, male).

$46 \%$ answered negatively to the fact that Facebook has access to their activities when they use this social network [Q12] and 54\% answered negatively to the off-Facebook activity [Q13]. "I have many concerns regarding the Facebook algorithm, generally, it makes me fill violated, I don't like the fact that it asks me so many personal questions, shows me ads and sends notifications that all related to my online activities. It seems that it collects everything about me, and I don't know why." (interviewee 19, female). In other hand, "I have nothing to hide so I don't care who accesses my information". (interviewee 21, male). Although $68 \%$ are aware of the consequences of being tracked online [Q15], 62\% have concerns regarding privacy or ethical issues [Q16].

There seems to be a relation between feeling nervous by seeing the online ads and feeling manipulated by the advertiser [Q23-24]. "Definitely, I don't like to be tracked by Facebook. . it is a little bit scary". "I feel awkward sometimes when I see ads related to my previous browsing." (interviewee 22, female) Similarly, "Marketers manipulate people by brain-washing techniques (i.e. offers, scarcity messages)". (interviewee 19, female) "Although I have been setting off everything on Facebook etc I still feel tracked and I don't like it because it seems to control my needs in a way". (interviewee 48, male) (Interviewee 40, male) believes that the internet censorship and online ads are related, and they limit our freedom in a way that is scary (Table 4 ).

Also, study participants who have no concerns regarding privacy or ethical issues [Q16] seem to like that Facebook picks and chooses the ads it thinks users will be interested in seeing [Q11]. "I will interact with online ads only if they are interested to me" (interviewee 21, male). "I have some concerns regarding privacy or ethical issues on Facebook. I would prefer if I could have full control of what I see there without giving my personal information". (Interviewee 22, female) (Table 4). Moreover, people who feel manipulated by the advertiser [Q23] feel that their personal information is taken without permission [Q22]. "I would prefer if I could have account with not sharing my information". (interviewee 19, female) "I believe that people who trust Facebook and other platforms do not give an appropriate attention to the small text that says to you to consist to any sharing of your personal data." (interviewee 48, male) "Sometimes, I am just afraid that one day Facebook will know my needs better than I know." (interviewee 19, female) (Table 4).

RQ2. How do consumers perceive and respond to Scarcity in algorithmically mediated digital marketing?

$72 \%$ of study participants believe that there are many advertisements online with scarcity phrases [Q16]. All interviewees believe that people are bombarded 
Table 4. Result Details RQ1.

\begin{tabular}{|l|l|l|}
\hline Items & rs & p (2-tailed) \\
\hline Q24-23 & 0.36856 & 0.00845 \\
Q16-11 & 0.33204 & 0.01849 \\
Q23-22 & 0.63174 & 0 \\
\hline
\end{tabular}

with ads with scarcity phrases. There seem to be a relation between people who tend to ignore scarcity phrases [Q18] and people who don't trust the online ads with offers [Q21]. "Lately, I don't trust the offers because I believe marketers try to manipulate people by this way so if I want to buy something I check it twice before any purchasing". (interviewee 22, female). "Most of online ads I see have scarcity messages. Several times I have been trusted these messages, when I realized that there are many tricks (prices vs quantity). It's really annoying." (interviewee 19, female). In other hand, "I like and trust online ads with offers mostly from trustworthy companies like Amazon or Zara". (interviewee 48, male)(Table 5).

Q20-30, 31, 32, 34 The experiment showed that the study participants who like tailored ads don't want to run the risk missing out on offers [30], they are upset if they miss buying some products of interest [31], they become frustrated when they are unable to get their preferred choice [32] and feel guilty that they can't control their spending [34]. "I like offers only when I am looking for a specific product and I would be upset if miss them out". (interviewee 22, female). "I like online shopping, but unfortunately it hides many traps." (interviewee 21, male). Similarly, "I like ads and ads with offers, I enjoy mostly 'online window shopping' with no purchasing". (interviewee 48, male) In other hand, "I feel insecure when I see tailored ads since I like shopping and sometimes, I buy things that I regret after. So, I cannot control my spending especially if I see things that I like." (interviewee 19, female). (Interviewee 40, male) believes that tailored ads are helpful, and one can benefit but only when having control over them; otherwise, it is "annoying" (Table 5).

Table 5. Result Details RQ2.

\begin{tabular}{|l|l|l|}
\hline Items & rs & p (2-tailed) \\
\hline Q18-21 & 0.40163 & 0.003845 \\
Q30-31 & 0.29963 & 0.03452 \\
Q20-31 & 0.42467 & 0.00211 \\
Q20-32 & 0.39759 & 0.00425 \\
Q20-34 & 0.33233 & 0.01838 \\
\hline \hline
\end{tabular}

RQ3. What are users' perceptions about the fairness in use of scarcity in algorithmic micro-targeting? 
Q19-23 There seem to be a relation between people who find that the products of their interest are often scarce [19] and people who feel manipulated by the advertiser [23]. "I know that marketers use sales tools such as scarcity messages .... although I feel triggered to see more details it doesn't mean that I will purchase the product. In other words, the scarcity message will not affect my decision to buy". (interviewee 48, male). "I believe that marketers sometimes create fake scarcity messages/offers to make more sales. It makes me feel insecure etc." (interviewee 22, female) Similarly, "There are people who have different weaknesses (shopaholic or in depression), and marketers use these people to gain money in some way." (interviewee 19, female). (interviewee 40, male) believes that there is a current need to protect people with shopping weaknesses and to provide them (for example technology education, training etc) (Table 6).

Q26-24 58\% of study participants think that it is not fair to use scarcity to convince them to purchase the product [26] and it seems to be related to people who got nervous by seeing the online ads [24]. "It is not fair to use scarcity, especially in the case of people with weaknesses." (interviewee 19, female). In other hand, "It is fair to use scarcity phrases only if they are not fake or misleading." (interviewee 48, male). Similarly, (interviewee 40, male) believes that it is totally unethical to create intentionally the misleading scarcity messages and there is a need to control these issues. "I can understand that marketers try to use different sale techniques and to stimulate their customers' needs, but ethical limitations definitely should be applied." (interviewee 40, male)(Table 6)

Q25-21 Moreover, people who feel that marketers intentionally created scarcity [25] seem to don't trust the online ads with offers [21]. "I don't trust ads with offers I believe most of them are fake or spam" (interviewee 21, male). "Usually, if I click to ad with offers or scarcity message and I am going to buy the product I try to read customers' reviews before purchasing" (interviewee 22, female). "Since I see ads based on my previous browsing and they have scarcity messages or offers on them, I believe that they are fake or intentionally created. Most of times I just ignore them." (interviewee 40, male) (Table 6).

Table 6. Result Details RQ3.

\begin{tabular}{|l|l|l|}
\hline Items & rs & p (2-tailed) \\
\hline Q19-23 & 0.44122 & 0.00134 \\
Q26-24 & 0.39622 & 0.00439 \\
Q25-21 & 0.40083 & 0.00392 \\
\hline
\end{tabular}




\section{Discussion}

The paper contributes to digital marketing theory by developing an awareness of the crucial link between cognitive marketing and understanding of fairness in algorithmic processes since that may affect consumers' and marketers' behaviour. It was revealed that whereas most people like the way how the algorithm of Facebook or any other similar platform works they have concerns regarding privacy or ethical issues and they feel violated and no control over the algorithmic processes and personal data. Also, people who don't trust the online ads with offers tend to ignore scarcity phrases. On the other hand, people who like tailored ads don't want to run the risk missing out on offers and they become frustrated and feel guilty that they can't control their spending. Also, people who find that the products of their interest are often scarce feel manipulated by the advertiser. Moreover, people who feel that marketers intentionally create scarcity (64\%) seem to don't trust the online ads with offers. The majority thinks that it is not fair to use scarcity to convince people to purchase the product and it makes them feel nervous by seeing the online ads. Moreover, people believe that marketers create misleading scarcity messages, and in their opinion, it is unethical. Another conclusion of our study is that people even who like online ads tailored to their preferences don't give attention to ad banners or to scarcity messages and don't have immediate desire to buy.

There doesn't appear to be a strong relation between participants liking online ads tailored to their preferences and an immediate desire to buy something $(\mathrm{rs}=0.20622, \mathrm{p}(2$-tailed $)=0.15076)$. The below heatmap, which is a data visualization technique, uses a warm-to-cool colour spectrum to show which parts of a page receive the most attention. Specifically, it shows that the study participants looked mostly on the map image of the top page and mostly ignored the ad banners. Also, ads got no clicks. "I interact with ads only when I am going to buy something". (interviewee 40, male). "Usually, I give attention to ads when I have time and mood." (Interviewee22, female) "Ad banners are so common, people don't give attention to them, especially if they just interested to read an article they will not interact with ads". (Interviewee 48, male) This phenomenon known as 'Banner blindness' and it could an instance of selective attention. According to (Kara Pernice, 2018) people direct their attention only to a subset of the stimuli in the environment - usually those related to their goals (i.e. read the article). Also, according to (Interviewee 48, male) "Ad banners with offers that are not eye-catching and not branding are not effective".

\section{Limitations \& Conclusions}

There are a number of limitations associated with this study. First, the sample is small in size and it mostly consists of undergraduate students. Second, there 
Fig. 1. The heatmap.

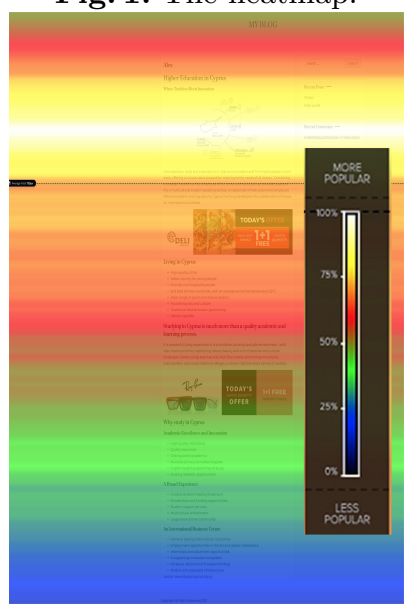

is a chance of recall bias in the process of gathering data since the participants were directed to the specific website instead of browsing on their own. Since marketers expect consumers to enjoy their marketing efforts and at the same time consumers expect marketers not only to anticipate their needs, but to be accountable for the ads content and techniques they use, there is a need to continue exploring and monitoring the fairness perceptions and beliefs of not only the users but also other stakeholders involved, such as marketers, algorithmic mediators, and government regulators. Finally, future studies will need target a larger sample of participants allowing the collection of more reliable data.

\section{Acknowledgements}

This project is partially funded by the European Union's Horizon 2020 research and innovation programme under grant agreement No. 810105 (CyCAT).

\section{References}

1. Aggarwal, P., Jun, S. Y., \& Huh, J. H.: Scarcity Messages. J. of Advertising, 40(3), 19-30. (2011)

2. Bayer, Judit, (2020). "Double harm to voters: Data-driven micro-targeting and democratic public discourse," Internet Policy Review: J. on Internet Regulation.

3. Becker, M. (2019). Privacy in the digital age: comparing and contrasting individual versus social approaches towards privacy. Ethics Inf Technol 21, 307-317.

4. Broeder, P., \& Derksen, R. (2018). Exclusivity in online targeted promotions: crosscultural preferences of consumers. International Journal of Business and Emerging Markets, 10(4), 396-408. 
5. Brock. T.C., (1968). Implications of Commodity Theory for Value Change. Psychological Foundations of Attitudes, 243-275.

6. Cialdini, Robert B. (2008). Influence: Science and Practice, Allyn \& Bacon: 4th Ed.

7. Dignum, V. (2018). Ethics in artificial intelligence: introduction to the special issue. Ethics Information Technology, 20(1), 1-3.

8. Donghee Shin, Yong Jin Park: Role of fairness, accountability, and transparency in algorithmic affordance. Comput. Hum. Behav. 98: 277-284 (2019); 2018.

9. Fischer, G. (2001). User Modeling and User-Adapted Interaction 11, 65-86.

10. Fromkin, H. L. (1970). Effects of experimentally aroused feelings of undistinctiveness upon valuation of scarce and novel experiences. J. of Personality \& Soc. Psych.

11. Gierl, H., \& Huettl, V. (2010). Are scarce products always more attractive? The interaction of different types of scarcity signals with products' suitability for conspicuous consumption. International Journal of Research in Marketing, 27(3), 225-235.

12. Gupta, S., Melewar, T.C. and Bourlakis, M.A. (2013), "A relational insight of brand personification in business-to-business markets", J. of General Management.

13. Howard, P. (2006). New Media Campaigns and the Managed Citizen (Communication, Society and Politics). Cambridge: Cambridge University Press.

14. Kleinberg, J., Ludwig, J., Mullainathan, S., Sunstein, C.R. Discrimination in the Age of Algorithms, Journal of Legal Analysis, Volume 10, 2018.

15. Khoa Trinh., A. (2014). Scarcity Effects on Consumer Purchase Intention in the context of E-commerce. Aalto University

16. Lanzing, M. (2016). The transparent self. Ethics Inf Technol 18, 9-16

17. Lee, M. K. (2018). Understanding perception of algorithmic decisions: Fairness, trust, and emotion in response to algorithmic management. Big Data \& Soc., 5(1).

18. Lepri, B., Oliver, N., Letouzé, E. et al. (2018). Fair, Transparent, and Accountable Algorithmic Decision-making Processes. Philos. Technol. 31, 611-627

19. Lynn, M. (1991). Scarcity effects on value: A quantitative review of the commodity theory literature. Psychology \& Marketing, 8, 43-57.

20. Manyika, J., Silberg, J., Presten, B. (2019). What Do We Do About the Biases in AI?. Harvard Business Review.

21. Mittone, L., \& Savadori, L. (2009). The scarcity bias. Applied Psychology: An International Review, 58(3), 453-468. Open J. of Business and Management, 2015, 3, 96-108Published Online January 2015 in SciRes.

22. Mussomeli, A., Neier, M., Takayama, B., Sniderman, B., \& Holdowsky, J. (2019). Building a cognitive digital supply network. Augmenting automation in an AI world. Deloitte Insights.

23. O'Neil, C. (2016). Weapons of math destruction: How big data increases inequality and threatens democracy (First edition.). New York: Crown.

24. Schumpe, B., Erb., H. (2015). Humans and uniqueness Science Progress.

25. Shi, Xiaohui \& Li, Feng \& Chumnumpan, Pattarin. (2020). The use of product scarcity in marketing. European Journal of Marketing. ahead-of-print.

26. Shin, Donghee \& Park, Yong Jin. (2019). Role of Fairness, Accountability, and Transparency in Algorithmic Affordance. Computers in Human Behavior.

27. The IEEE Global Initiative on Ethics of Autonomous and Intelligent Systems. Ethically Aligned Design: A Vision for Prioritizing Human Well-being with Autonomous and Intelligent Systems, First Edition. IEEE, 2019.

28. Wing, Jeannette M. "Data for Good: FATES, Elaborated" Columbia University Data Science Institute, Jan. 23, 2018

29. Winter, S., Maslowska, E., Vos, A., (2021). The effects of trait-based personalization in social media advertising 